\section{CASE OF ACUTE INTESTINAL OBSTRUCTION} IN A HAEMOPHILIC.

By B. H. KINGSFORD, M.D.LoND., WOKING.

AN ileal variety of intussusception (which according to Leichenstern occurs in only 8 per cent. of cases) complicated by containing a Meckel's diverticulum, and occurring in a haemophilic, forms a combination sufficiently rare to merit recording:

At 8 p.m., November 24th, 1905, I was called to see a boy, aged 10, who had been ill since the previous day, with what was thought to be a "bilious attack," to which he was said to be subject.

\section{Family History.}

His maternal great-grandmother was healthy, but both he sisters died from haemorrhage in confinements, and both he -brothers died from haemorrhage. She had five children-fou daughters, one of whom died from haemorrhage in her sixth confinement, and another died from " change of life" ; and one son (patient's grandfather), who, during the first thirty years of life, was a "bleeder.". He had one son and three daughters (on of whom was patient's mother), none of whom suffered from haemophilia. The patient had one brother, a marked haemochilic, and one sister, healthy.
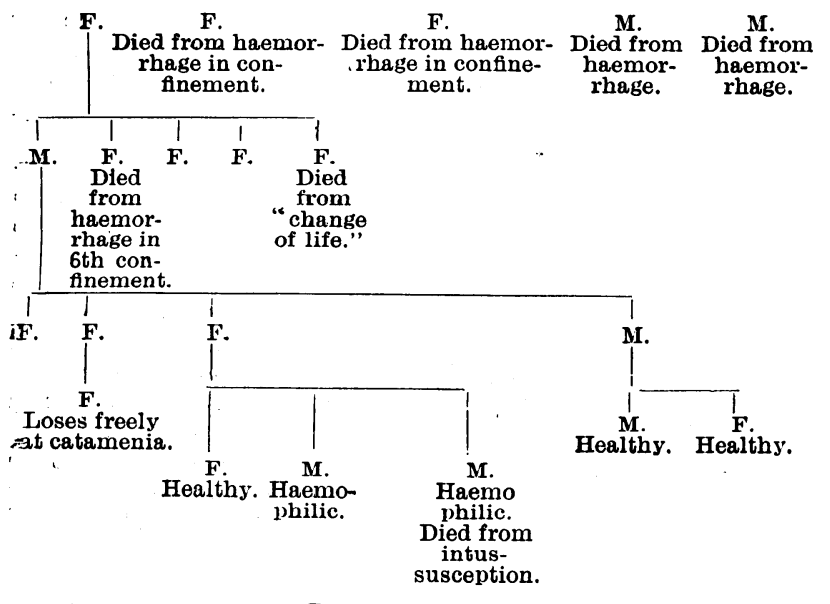

Personal Histor

Apart from haemophilia, he had had good health. He was subject to haemorrhagic joints, had bled for days after tooth extraction, and nearly died from haemorrhage after circum cision.

\section{Present Illness.}

The bowels were moved early on the previous day, but for more than twenty-four hours there had been absolute constipation, some slight abdominal pain, and frequent vomiting.

\section{Condition on Examination.}

The temperature was $99^{\circ} \mathrm{F}$., and pulse 84 . The tongue was furred. There was some bleeding from the gums, due to tooth eruption. The abdomen moved fairly well during respiration, but movement was restricted over lower part; there was no distension. Beneath the right rectus muscle, just above it insertion into the pubes, there wectume tion ind in the pubes, there was some resistance to palpitation, and on deep pressure a fullness could be detected, and some tenderness elicited. Over the same area there was sligh impairment of resonance. Rectum empty ; in the right anterio quadrant there was a smooth, elastic bulging, apparently part of a mass, the size of a hen's egg. Micturition was frequent, but not painful ; urine acid, no albumen or sugar.

\section{Progress.}

On the following day the temperature was $99^{\circ} \mathrm{F}$. and the pulse 96. Vomiting continued at variable intervals but much less frequently, though more copiously, than on previous day; the bowels had not moved; enemata returned unchanged. During the night there was passed a copious chocolate-coloured evacuation consisting of altered blood. Physical signs unchanged, but more pronounced. Th bulging per rectum had increased. Dr. A. E. Garrod saw the case in consultation with me. During the early part of November 26 th there was nausea, but no vomiting In the afternoon vomiting returned, and in the frequent and pin becme more frequin and pase in the rose half a degree. On November 27 th, about 4 a.m., he suddenly rose half a degree.

Post-mortem Examination Sixteen Hours after Death.

Beneath the right rectus and lying mainly in the pelvis was large irreducible ileal intussusception about $5 \mathrm{in}$. long and involving more than $1 \mathrm{ft}$. of bowel ; the distal extremity was about 3 in from the caecum. Lying in contact with its posterior surface 3 was a long vermiform appendix dipping into the pelvis. Protruding from the intussusception was the blind end of a long Meckel's diverticulum. On laying open the intussusception this diverticulum was found to open into the inner tube of the ileum a short distance from the apex.

The family history presents the following noteworthy points :

1. At least three female members of the family died from haemorrhage.

2. The hereditary taint was transmitted through a male haemophilic of the second generation, though in a weakened form, as his immediate offspring (third generation) were exempt, but reappeared in the fourth generation through the female.

The exact diagnosis of the abdominal condition was beset with difficulties. At first the signs suggested inflammation of a long appendix dipping into the pelvis, and it is interesting to note the position of the appendix as found post mortem. The personal history suggested paralysis of bowel due to a haemorrhage into the intestinal wall or mesentery.

The absence of tenesmus and of severe spasmodic pain so characteristic of intussusception, and the difficulty of determining the outline of the tumour, owing to its lying in the pelvis behind the pubes, made it impossible to diagnose an intussusception with certainty. The blood passed by the rectum was of the character of chronic rather than acute intussusception, and in this patient was of doubtful diagnostic value.

Laparotomy as a means of diagnosis or of treatment was considered unjustifiable.

Whether the presence of a Meckel's diverticulum was the cause of the lesion or a coincidence is not quite clear.

I incline to the view that it was indirectly the cause.

One would expect the diverticulum to be at the apex of the intussusception instead of a short distance from it. But this is analogous to an intestinal polypus which is an acknowledged cause of the lesion. Though usually found at the apex, occasionally a polypus is some distance from it owing to shifting of the entering and returning layers.

Treves ${ }^{1}$ mentions two specimens in which a small and short Meckel's diverticulum had become inverted so as to project into the lumen of the bowel and so cause intussus. ception. Dobson ${ }^{2}$ describes a similar case and has collected thirteen cases of this nature. Carwardine ${ }^{3}$ describes a triple telescopic intussusception with an inverted diverticulum. Golding-Bird 4 relates a case of intussusception into a diverticulum, and two similar cases have been described by Parker and Southall. ${ }^{5}$

In my case there was no inversion of the diverticulum.

But it seems reasonable to suppose that irritation in a Meckel's diverticulum or in the portion of bowel from which it springs is more likely to set up irregular peristalsis than in a normal bowel; and the cases quoted above, I think, tend to support this view.

The position and irreducibility of the obstruction proves that treatment by injection or inflation would have been useless.

$$
\text { REFERENCES. }
$$

1 Treves, Intestinal Obstruclion. ${ }_{2}$ Lancet, April 25th, 1903. 3 Ibid. February 20th, 1904. ${ }^{4}$ Trans. Clin. Soc., 1896. ${ }^{5}$ Lancet, November 12th, 1904.

\section{SOME POINTS IN \\ THE ANATOMY AND PATHOLOGY OF THE HERNIAL SAC.}

By E. SCOTT CARMICHAEL, M.B., F.R.C.S.E,, ASSISTANT SURGEON, ROYAL HOSPITAL FOR SICK CHILDREN, EDINBURGH.

IT is the purpose of this paper to record certain observa tions on the hernial sac, gained by an experience of a large number of cases examined before operation, and from the anatomical and pathological conditions found in over 300 operations of radical cure for hernia, chiefly in children. The observations were made in the out-patient children. of the hospital and in the operating theatre, as well as on surgically healthy children in the medical wards. A routine examination was made of children admitted to the hospital for medical ailments, and not subjected to surgical treatment afterwards.

The etiology of the hernial sac has of late been much under discussion, and attention has been drawn to the 